http://jmscr.igmpublication.org/home/ ISSN (e)-2347-176x ISSN (p) 2455-0450

crossref DOI: https://dx.doi.org/10.18535/jmscr/v7i11.92

\author{
Journal Of Medical Science And Clinical Research \\ IGM Publication \\ An Official Publication of IGM Publication
}

\title{
Porto Pulmonary Syndrome in Children
}

\author{
Author \\ Sujay Chaudhuri \\ Division of Pediatric Gastroenterology, Department of Gastroenterology, PGIMER Chandigarh \\ *Corresponding Author \\ Sujay Chaudhuri
}

\begin{abstract}
Background: Porto pulmonary hypertension $(\mathrm{PoPH})$ is combination of portal hypertension and pre capillary pulmonary arterial hypertension (PAH). Prognosis is poor in pediatric patients. To evaluate incidence, clinical profile, investigational profile and outcome of pediatric PoPH, the following study was done.

Method: The children (age 3 - 12 years) who had portal hypertension and respiratory distress were admitted in pediatric gastro enterology ward of PGIMER - Chandigarh from July 1993 to June 2003. History and clinical examination (Ascites, GI Bleed, Jaundice, dyspnea, fatigue, chest pain, syncope etc.), investigations (USG whole abdomen, LFT, UGI Endoscopy, pulse oxymetry, echocardiography, contrast echocardiography, chest $X$-ray etc.) were noted.

Simplified Bernoulli equation is used to detect PAP by echocardiography. Contrast echocardiography is done to rule out hepato pulmonary syndrome.

Results: Out of 10 cases of portal hypertension, 6 were male, 4 were female. Age was 3 - 12 years (mean 8 year). Out of 10 cases, 6 were cirrhosis, 4 were non cirrhotic portal hypertension. Invasive procedure like cardiac catheterization was not done. PAP (measured by echocardiography) was $40-55 \mathrm{~mm} \mathrm{Hg.} 4$ patients who had non cirrhotic portal hypertension improved after portal systemic shunt and reduction of portal hypertension. 6 patients who had cirrhosis and pulmonary hypertension could not survive. Symptomatic treatment of pulmonary hypertension was done. (IV prostaglandins etc.)

Conclusion: Porto pulmonary hypertension is a rare but serious problem in children. Research is still going on to get best management.

Keyword: Porto Pulmonary Syndrome in Children.
\end{abstract}

\section{Introduction}

Mantz and Craig reported a case called porto pulmonary (PoPH) syndrome in 1951, where there is combination of portal hypertension and pulmonary hypertension. Mean pulmonary arterial hypertension (mPAP) remains > $25 \mathrm{~m} \mathrm{Hg}$, pulmonary capillary wedge pressure $(\mathrm{PCWP})<15$ $\mathrm{mm} \mathrm{Hg}$ and pulmonary vascular resistance (PVR) $>3$ wood units $(\mathrm{WU}){ }^{(1)}$
Histopathology of pulmonary artery in porto pulmonary syndrome is similar to that of idiopathic pulmonary hypertension. Findings are vasoconstriction, endothelial and smooth muscle proliferation, plexogenic arteriopathy, in situ thrombosis and fibrosis. ${ }^{(2)}$

In adults with liver cirrhosis, incidence of porto pulmonary hypertension $(\mathrm{PoPH})$ is reported to be $2-8 \%{ }^{(3)}$ 
Various studies have shown that patients with PoPH have poorer survival rate and all cause hospitalization rates compared to that with Ideopathic pulmonary arterial hypertension (IPAH). ${ }^{(4)}$

A diagnosis of PoPH in a cirrhosis is associated with high mortality even after liver transplantation. PoPH may be a contraindication to liver transplantation. ${ }^{(5)}$

Adult patients with $\mathrm{PoPH}$ are recorded in literature in most cases. But pediatric $\mathrm{PoPH}$ are scanty in literature. In an autopsy series of children with portal hypertension, 5.4\% had histologic evidence of pulmonary arterial hypertension. $^{(6)}$

$\mathrm{PoPH}$ is disorder which has high mortality in pediatric patients. Due to limited experience, there is under recognition and delayed diagnosis. Condino et al reported high mortality in a series of 7 patients where 4 (4/7) died. ${ }^{(7)}$

Syncope is most common presenting symptom of $\mathrm{PoPH}$ in children. Risk of death is three fold higher in pediatric PoPH than IPAH patients. ${ }^{(8)}$

In adult series, alcoholic liver disease and hepatitis are common cause of cirrhosis. ${ }^{(9)}$

In children with PoPH, congenital causes of liver cirrhosis are more common (e.g. Billiary atresia) but in pediatric PoPH, there are cases where there is portal hypertension without cirrhosis (porto systemic shunt) ${ }^{(10)}$

Porto systemic shunt and hepatic failure leads to increased circulating vasodilators. ${ }^{(11)}$

This condition leads to splanchnic vasidilation and low systemic vascular resistance. ${ }^{(12)}$

Volume overload into splanchnic vasculature leads to bowel wall congestion and liberation of endotoxins and cytokines. In addition, higher cardiac output and increased flow within pulmonary vasculature causes increased shear forces and increased mPAP (mean pulmonary artery pressure). ${ }^{(13)}$

Pulmonary vasculature's response to hyper dynamic state is variable. A vasodilatory response may lead to hepato pulmonary syndrome. Vasoconstriction may lead to PVD (pulmonary vascular dilatation) similar to precapillary $\mathrm{PH}$ (pulmonary hypertension).

Genetic and environmental factors determine whether vasodilation and hepato pulmonary syndrome will occur or vasoconstriction and PoPH will occur. ${ }^{(13)}$

Ecochard et al observed that hepato pulmonary syndrome can precede to development of PoPH. Hypoxia secondary to hepato pulmonary syndrome can lead to PoPH. ${ }^{(14)}$

In both pediatric and adult $\mathrm{PoPH}$, histopathological changes are similar to that of IPAH. ${ }^{(15)}$

PAH occurs due to dys-regulation of vasodilators and vasoconstrictors including prostacyclin, nitric oxaide, endothelin-I, thromboxam $\mathrm{A}_{2}$ and serotonin. ${ }^{(16)(17)}$

Due to release of inflammatory mediators, there is continued increase to pulmonary blood flow, sheer stress on pulmonary vascular bed, subsequent vasoconstriction and pulmonary vascular remodeling. In portal hypertension, there is porto systemic shunting and inflammatory mediators escape metabolic degradation by liver. Then they directly act upon pulmonary vasculature. ${ }^{(18)(19)}$

There is no association between severity of portal hypertension and severity of PAH (based on right heart hemodynamic measurements). ${ }^{(18)}$

The most effective therapy for PoPH has not yet established. The use of prostanoids, calcium channel bluchers, endothelin receptor antagonist, phosphodiasterase - 5 (PDE 5) inhibitors have been mentioned. In a study from Mayo Clinic, researchers observed that medical therapy alone or medical therapy plus liver transplant improved survival. $^{(20)}$

No single modality of treatment has shown superiority over others. Treatment with continuous intravenous epoprostenal has been associated with hypersplenism. ${ }^{(21)}$

\section{Method}

The children (age 3 - 12 years) who had portal hypertension and respiratory distress were admitted in pediatric gastro enterology ward of 
PGIMER - Chandigarh from July 1993 to June 2003. History and clinical examination (Ascites, GI Bleed, Jaundice, dyspnea, fatigue, chest pain, syncope etc.), investigations (USG whole abdomen, LFT, UGI Endoscopy, pulse oxymetry, echocardiography, contrast echocardiography, chest X-ray etc.) were noted.

Simplified Bernoulli equation is used to detect PAP by echocardiography. Contrast echocardiography is done to rule out hepato pulmonary syndrome.

\section{Results}

Out of 10 cases of portal hypertension, 6 were male, 4 were female. Age was $3-12$ years (mean 8 year). Out of 10 cases, 6 were cirrhosis, 4 were non cirrhotic portal hypertension. Invasive procedure like cardiac catheterization was not done. PAP (measured by echocardiography) was $40-55 \mathrm{~mm} \mathrm{Hg} .4$ patients who had non cirrhotic portal hypertension improved after portal systemic shunt and reduction of portal hypertension. 6 patients who had cirrhosis and pulmonary hypertension could not survive. Symptomatic treatment of pulmonary hypertension was done. (IV prostaglandins etc.)

\section{Discussion}

Splanchnic blood flow is increased in portal hypertension secondary to increase of local and systemic vasodilators. It is potentiated by use of prostacyclin therapy for porto pulmonary hypertension $(\mathrm{PoPH}){ }^{(12)}$

Various studies have evaluated utility of screening all liver cirrhosis patients with echocardiography to detect PoPH. Though echocardiography is useful for screening at risk population, it is insufficient to evaluate PoPH. Cardiac catheterization and targeted medical therapy with closure of intrahepatic shunts should be considered before liver transplantation. ${ }^{(22)}$

In our series of 10 cases of PoPH, 6 had cirrhosis and 4 had non cirrhotic portal hypertension cirrhotic patients could not survive but 4 patients of non cirrhotic portal hypertension improved with treatment.

Though PoPH is diagnosed $4-7$ years after diagnosis of portal hypertension diagnosis of $\mathrm{PoPH}$ is actually made during hemodynamic monitoring before liver transplantation. ${ }^{(20)}$

Diagnosis of PoPH is made on basis of hemodynamic criteria where patients with portal hypertension and or liver disease (ascites / varices / splenomegaly) have mean pulmonary artery pressure MPAP > $25 \mathrm{~mm}$ at rest / pulmonary vascular resistance - PVR > 240 dynes / pulmonary artery occlusion pressure PAOP $<15$ $\mathrm{mm}$ or transpulmonary gradient TPG $>12 \mathrm{~mm}$ where TPG $=$ MPAP - PaoP. ${ }^{(23)}$

Symptomatic patients of $\mathrm{PoPH}$ present with right heart dysfunction secondary to pulmonary hypertension and its consequent dyspnea, fatigue, chest pain and syncope. ${ }^{(24)}$

Sixty percent of patients with PoPH have stage III - IV NYHA heart failure. ${ }^{(25)}$

$\mathrm{PoPH}$ is independent of severity of cirrhosis but may be more common in specific types of cirrhosis. Some studies have mentioned that PoPH is more common in auto immune hepatitis related cirrhosis but less in hepatitis $\mathrm{C}$ cirrhosis. ${ }^{(26)}$

In $\mathrm{PoPH}$ there is imbalance between vasoconstrictors and vasodilator substance. Due to liver cirrhosis, toxic (vasoconstrictors) substances escape metabolism bypassing liver. Moreover, a key pathogenic factor for declining of PoPH status is cirrhotic cardiomyopathy with myocardial thickening and diastolic dysfunction. ${ }^{(27)}$

One autopsy series have shown that cirrhotic patients have thickened pulmonary arteries. ${ }^{(26)}$

Calcium channel blocker, beta blockers, nitrates are all tried but most potent and widely used substances are prostaglandin (and prostacyclin) analogs, phosphodiasterase inhibitors, nitric oxide and most recently, endothelin receptor antagonist. Inhaled nitric oxide (i No) vasodilates, decreases PAP and PVR without affecting systemic artery pressure. It is rapidly inactivated by hemoglobin. It improves oxygenation by redistributing pulmonary blood flow in ventilated area. But i No 
requires intubation, it cannot be used for long period due to methenaglobinemia. ${ }^{(29)}$

Prostaglandine like PGE1 (Alprostadil), PGE2 (Prostacyclin) and more stable, long acting Epoprostenol (Iloprost) are tried. Iloprost can lower PAP by $29-46 \%$ and PVR by $21-71 \%$. (30)

Phosphodiesterase inhibitors like milrinone causes selective pulmonary vasodilatation, increases myocardial contractile force, increase extent of relaxation. ${ }^{(31)}$

Endothelin I receptor antagonist (Busentan) improves oxygenation and PVR. ${ }^{(32)}$

Imatinib (designed to treat chrome myeloid leukemia) has been shown to reverse pulmonary remodeling associated with $\mathrm{PoPH}{ }^{(33)}$

\section{Conclusion}

Porto pulmonary hypertension is a rare but serious problem in children. Research is still going on to get best management.

\section{Conflict of interest - Nil}

\section{References}

1. Mantz F et al, Portal axis thrombosis with spontaneous portacaval shunt and resultant cor pulmonale AMA Archives of pathology 1951, 52 (1) 91-97 (Pub Med) (google scholar).

2. Krowka MJ et al, A spectrum of pulmonary vascular pathology in porto pulmonary syndrome. Liver transplant 2000, 6 (2) 241-242, (Pub Med) (google scholar).

3. Lebrec D et al, Pulmonary hypertension complicating portal hypertension : prevalence and relation to splanchmic hemodynamic Gastroenteral 1991, 100 : 520 - 528, (Pub Med) (google scholar).

4. Krowka MJ et al, Porto pulmonary hypertension a report from US based reveal registry. Chest 2012141 (4), 906 915, (Pub Med) (google scholar).
5. Krowka MJ et al, Pulmonary hemadynamic and perioperative cardiopulmonary related mortality in patients with porto pulmonary hypertension undergoing liver transplantation. (Liver Transplantation) 2000, 6 (4), 443 - 450. (Pub Med) (google scholar).

6. Ridaura - Sauz c et al, Porto Pulmonary hypertension in children. A study in pediatric autopsies. Arch Med Res 2009, 40 (7), 635-639. (Pub Med) (google scholar).

7. Condino A A et al, Porto pulmonary hypertension in pediatric patients. $\mathrm{J}$ pediatr 2005147 (1) 20-26. PMC free ar.

8. Kawnt SM et al, Hemodynamics and survival of patients with porto pulmonary hypertension. Liver transplant 2005. 11 (9), 1007-1111.

9. Ctton CL et al, Role of echocardiography in detecting porto pulmonary hypertension in liver transplant candidates. Liver transplant 2002, 8 (11), 1051-1054. (Pub Med) (google scholar).

10. Cohen MD et al, Primary pulmonary hypertension an unusual case associated with extra hepatic portal hypertension. Hepatology 19833 (4) 588-592. (Pub Med) (google scholar).

11. Bolognesi $\mathrm{M}$ et al, Splanchnic vasodilatation and hyper dynamic circulating syndrome in cirrhosis. World $\mathbf{J}$ gastroenterol 2014, 20 (10), 2555-2563. (PMC free article) (Pub Med)

12. Martell $M$ et al, Physiopathology of splanchnic vasodilation in portal hypertension. World J Hepatol

20 (10) , 208-220. (PMC free article) (Pub Med) (google scholar).

13. Hoeper MM et al, Porto pulmonary hypertension and hepato pulmonary syndrome. Lancet 2004 , 363-9419, 1461 1468. (Pub Med) (google scholar). 
14. Ecochard Dugelay E et al, Porto pulmonary hypertension in liver disease presenting in childhood. $\mathrm{J}$ pediatr gastroenterol nutrition 2015, 61(3). 346354. (Pub Med) (google scholar).

15. Edwards BS et al, Coexistent portal and pulmonary hypertension: Morphologic and clinical feature. J AM coll cardiac 1987, 10(6), 1233-1238. (Pub Med) (google scholar).

16. Lourenco AP et al, Current patho physiological concepts and management of pulmonary hypertension. Int $\mathbf{J}$ cardiac 2012, 155(3) 350-361. (Pub Med) (google scholar).

17. Giaid A et al, Expression of endothelia I in lung of patients with pulmonary hypertension. N Eng J Med 1993, 328(24) 1732-1739. (Pub Med) (google scholar).

18. Benjaminov FS et al Porto pulmonary hypertension in decompensated cirrhosis with refractory ascites. Gut 2003 52(9) 1355-1362. (PMC free article) (Pub Med) (google scholar).

19. Humbert $M$ et al Increased Inter leukin 1 and inter leukin 6 serum concentrations in severe pulmonary hypertension. Am J respire crit care Med 1995 151(5), 16281631. (Pub Med) (google scholar).

20. Hadengue et al PH complicating portal hypertension. Prevalence and relation to splanchnic hemodynamic.

Gastroenterology 1991. 100 : 520-528.

21. Swanson KL et al Survival in porto pulmonary hypertension : Mayo clinic experience categorized by treatment subgroups. Am J transplant 2008, 8(11), 2445-2453. (Pub Med) (google scholar).

22. Touma W et al, Epoprostenol - induced hyper splenism in porto pulmonary hypertension. Am J Med Sci 2012, 344(5), 345-349. (Pub Med) (google scholar).
23. Torregrosa $M$ et al, Role of Doppler echocardiography in assessment of porto pulmonary hypertension in liver transplantation candidates., Transplantation 2001, 71(4) 572-574. (Pub Med) (google scholar).

24. Martinex Palli et al, Liver transplant in high risk patients, Transplant proceeding $200537: 3861$ - 3864

25. Le Pavec et al, Porto pulmonary hypertension : Survival and prognostic factors. Am J Resp crit Cave Med Vol 178 PP $637-6$.

26. Tapper EB et al, Porto pulmonary hypertension: Imatinib as a novel treatment and the Emory experience with this condition. Transplant proc 2009 June 41 (5) 1969 - 71.

27. Egermayer et al, Role of serotonin in acute and chronic pulmonary hypertension. Thorax 199954 : 161 - 168 .

28. Matsubara O et al, Histometrical investigations of pulmonary artery in severe hepatic disease. J Pathol, 1984143 $-31$

29. Stendel et al, Inhaled nitric oxide : Basic biology and clinical applications. Anesthesiology $199991: 1090$ - 121.

30. KUO PC et al, Continuous intravenous infusion of epoprostenol for treatment of porto pulmonary hypertension. Transplantation, $199763: 604$

31. Berkels et al, Modulation of human platelet aggregation by phosphodieshterase type 5 inhibitor sidenafil, J Cardiovasc Pharmacolo, 2001, 37 : 413 - 421

32. Hoeper et al, Bosentan therapy for porto pulmonary hypertension. Eur Respir J $2005,25: 502-8$

33. Ghofrani et al, Imatinib for treatment of pulmonary arterial hypertension. $\mathrm{N}$ Eng $\mathrm{J}$ Med 2005353 : 1412 - 1413. 